\title{
Evaluation of QTc Interval Prolongation in Breast Cancer Patients after Treatment with Epirubicin, Cyclophosphamide, and Docetaxel and the Influence of Interobserver Variation
}

\author{
Julian Puppe ${ }^{a, b} \quad$ Deborah van Ooyen $^{a, b} \quad J^{2}$ eanne Neise ${ }^{a, b}$ Fabinshy Thangarajah $^{a, b}$ \\ Christian Eichler $^{c}$ Stefan Krämer $^{a, b}$ Roman Pfister $^{d}$ Peter Mallmann $^{a, b}$ Marina Wirtz $^{a, b}$ \\ Guido Michels ${ }^{d}$ \\ ${ }^{a}$ Department of Gynaecology and Obstetrics, University Hospital Cologne, Cologne, Germany; \\ ${ }^{b} \mathrm{ClO}$ Center for Integrated Oncology Cologne/Bonn, Bonn, Germany; \\ ${ }^{\mathrm{c}}$ Breast Center, Holweide Hospital, Cologne, Germany; \\ ${ }^{\mathrm{d}}$ Department III of Internal Medicine, Heart Centre of the University of Cologne, Cologne, Germany
}

\section{Keywords}

Breast cancer · Chemotherapy · Anthracyclines .

Epirubicin · Cardiotoxicity · QT prolongation

\section{Summary}

Background: Chemotherapy with anthracyclines is associated with life-threatening electrocardiographic alterations including corrected QT (QTc) interval prolongation. Patients and Methods: In this study we assessed the effect of epirubicin, cyclophosphamide, and docetaxel (ECDoc) on the QTc interval in 10 patients with early breast cancer. Cardiac toxicity was assessed with symptoms, transthoracic echocardiography, electrocardiography (ECG), and serum cardiac markers at baseline and after 4 cycles of EC and 4 cycles of docetaxel. To evaluate the influence of interobserver variation, the QTc interval was analyzed by a cardiologist, a gynecologist, and with automated ECG interpretation software. Results: There was a significant QTc prolongation after EC treatment independent of the investigator. In addition, a significant increase in $\mathrm{N}$-terminal prohormone of brain natriuretic peptide (NT-proBNP) levels was noted after EC treatment. QTc prolongation and NT-proBNP levels normalized after docetaxel treatment. Other biochemical markers were within normal ranges. No clinically relevant effect on left ventricular ejection fraction was observed.

Julian Puppe and Deborah van Ooyen contributed equally to this article.
Conclusion: This prospective study demonstrated that EC treatment increases the QTc interval and NT-proBNP levels in women with early breast cancer. This effect was reversible and independent of docetaxel administration. Moreover, the treating physician can safely perform QTc interval evaluation as part of clinical routine independent of his/her specialty. Due to the small number of patients, further conclusions are limited at this point.

(c) 2017 S. Karger GmbH, Freiburg

\section{Introduction}

Anthracyclines play an important role in the neoadjuvant treatment of breast cancer patients [1]. However, these drugs are not free of adverse effects. Cardiovascular toxicity has gained increasing attention as an adverse event in cancer treatment. Anthracyclines such as epirubicin can cause left ventricular dysfunction [2, 3]. This has led to the regular testing of left ventricular ejection fraction (LVEF) by echocardiography for patients treated with anthracyclines [4]. In addition, serum biomarkers like cardiac troponin are widely used to detect cardiac damage [5]. However, the use of troponin as a prognostic tool in cancer therapy has been largely disappointing [6]. Natriuretic peptides, such as brain natriuretic peptide (BNP) and its aminoterminal component (NTproBNP) are used to diagnose acute and chronic heart failure [7].

\section{KARGER}

() 2017 S. Karger GmbH, Freiburg 
Table 1. Characteristics of the patient cohort

\begin{tabular}{ll}
\hline Characteristics & \\
\hline Patients, $\mathrm{n}$ & 10 \\
Age, years & $58.10 \pm 9.04$ \\
Weight, $\mathrm{kg}$ & $73.06 \pm 23.31$ \\
Body mass index & $26.20 \pm 8.26$ \\
Stage, $\mathrm{n}(\%)$ & \\
T1 & $5(50)$ \\
T2 & $2(20)$ \\
T3 & $0(0)$ \\
Missing & $3(30)$ \\
Nodal status, $\mathrm{n}(\%)$ & \\
Positive & $4(40)$ \\
Negative & $1(10)$ \\
Missing & $5(50)$ \\
Grading, $\mathrm{n}(\%)$ & \\
1 & $1(10)$ \\
2 & $5(50)$ \\
3 & $4(40)$ \\
Missing & - \\
Hormone receptor, $\mathrm{n}(\%)$ & \\
Positive & $7(70)$ \\
Negative & $2(20)$ \\
Missing & $1(10)$ \\
HER2 status, $\mathrm{n}(\%)$ & \\
Positive & $6(60)$ \\
Negative & $4(40)$ \\
Preexisting cardiac disease & hypertension \\
Cardiac medication & $(\mathrm{n}=3)$ \\
& $\mathrm{beta}$ blockers \\
$(\mathrm{n}=3)$ \\
\hline
\end{tabular}

Some studies describe a substantial increase in NT-proBNP in patients treated with anthracyclines with no effect on echocardiography or clinical outcome [8]. Other proposed markers such as creatine kinase $(\mathrm{CK})$, the cardiac-specific creatine kinase-MB (CK-MB), or C-reactive protein (CRP) have shown no clinical value so far [9].

Long QT syndrome is a repolarization disorder of the myocardium, which is characterized by a prolongation of the QT interval on electrocardiogram (ECG). This can be caused by drugs or hereditary ion channel abnormalities by blocking potassium channels or opening sodium or calcium channels [10]. Drug-induced QT interval prolongation has been identified as a further crucial risk factor for cardiac toxicity and is potentially leading to palpitations, syncope, and sudden cardiac death due to serious ventricular arrhythmias such as Torsade de Pointes [11, 12]. In clinical practice, a QTc interval (corrected for heart rate) of $>470 \mathrm{~ms}$ is associated with a 3 -fold risk increase for sudden cardiac death [13, 14]. Anticancer drugs might cause QT prolongation [15]. One retrospective study on childhood cancer survivors describes a persistent QT interval prolongation in 19 of 33 patients receiving anthracyclines (doxorubicin equivalent) [16]. A recent study investigated whether the free radical scavenger superoxide dismutase (SOD) protects against anthracycline-induced cardiotoxicity, and reported a prolongation of the QTc interval after doxorubicin plus cyclophospha- mide (AC) but no cardioprotective effect of SOD [17]. Another study in 34 patients demonstrated that exposure to epirubicin, cyclophosphamide, and 5-fluorouracil in women with breast cancer is correlated with QTc interval prolongation [18]. However, the influence of the widely used EC-Doc regimen (epirubicin, cyclophosphamide, and docetaxel) for breast cancer treatment on the QTc interval remains to be further elucidated. Therefore, we prospectively investigated the effect of EC-Doc on cardiotoxicity in women with early breast cancer.

The variability in ECG measurements and analysis between different investigators can be large [19]. In Germany, gynecologists generally administer breast cancer chemotherapy, and not all outpatient chemotherapy centers have direct access to a cardiology department. For this reason, we also evaluated if gynecologists can reliably produce and interpret ECG data as part of clinical routine and whether the use of automated ECG interpretation software can support QT interval interpretation.

\section{Patients and Methods}

\section{Patients}

A total of 10 women with early breast cancer were enrolled in this observational study and their data prospectively studied. All patients were treated in the Outpatient Chemotherapy Center at the Department Obstetrics and Gynecology, University Hospital Cologne. The study protocol was approved by the local ethics committee and accepted as an extended safety measure for chemotherapy treatment. Further patient characteristics are listed in table 1.

\section{EC-Doc Regimen}

The neoadjuvant regimen $(\mathrm{n}=8)$ consisted of 4 cycles of epirubicin $90 \mathrm{mg} / \mathrm{m}^{2}$ and cyclophosphamide $600 \mathrm{mg} / \mathrm{m}^{2}$, given on day 1 of a 14-day cycle. 2 patients were treated adjuvantly at the same dosage on day 1 of a 21-day cycle. Subsequently, 6 patients received 4 cycles of docetaxel $100 \mathrm{mg} / \mathrm{m}^{2}$ with $(\mathrm{n}=4)$ or without $(\mathrm{n}=2)$ trastuzumab $15 \mathrm{mg} / \mathrm{kg}$ body weight dependent on their HER2 status every 21 days. Data on docetaxel was missing for 4 patients.

\section{Electrocardiographic Assessments}

A standard 12-lead ECG was recorded before $(<20 \mathrm{~min})$ and immediately after the 4 th cycle of EC or the 4 th cycle of docetaxel $(<20 \mathrm{~min})$. The same welltrained personnel performed all ECGs. QT intervals were blinded for the treatment group and results of the computer software and independently analyzed by a senior cardiologist, a gynecologist, and with an automated ECG interpretation software (Schiller Cardiovit AT-10 plus, Felsberg, Germany). In order to reduce the dependence of QT interval on the heart rate, Bazett's formula was applied [20]. Experienced oncology nurses evaluated the patients for clinical symptoms of cardiotoxicity.

\section{LVEF Assessments}

The effect of EC on systolic heart function was investigated by echocardiography (LVEF) at baseline and within 14 days of EC or docetaxel treatment. The 2-dimensional echocardiographic analyses were performed according to the American Society of Echocardiography recommendations and with commercially available equipment (Philips IE33, Andover, MA, USA) featuring a 2-5 $\mathrm{MHz}$ transducer. LVEF was calculated with the Simpson's biplane method.

\section{Serum Cardiac Markers}

CK-MB and NT-proBNP were analyzed during routine blood tests within 7 days before the first cycle of EC and 14 days after the 4th cycle of EC or docetaxel. All analyses were performed at the Department of Clinical Chemistry of the University Hospital Cologne. 
a)

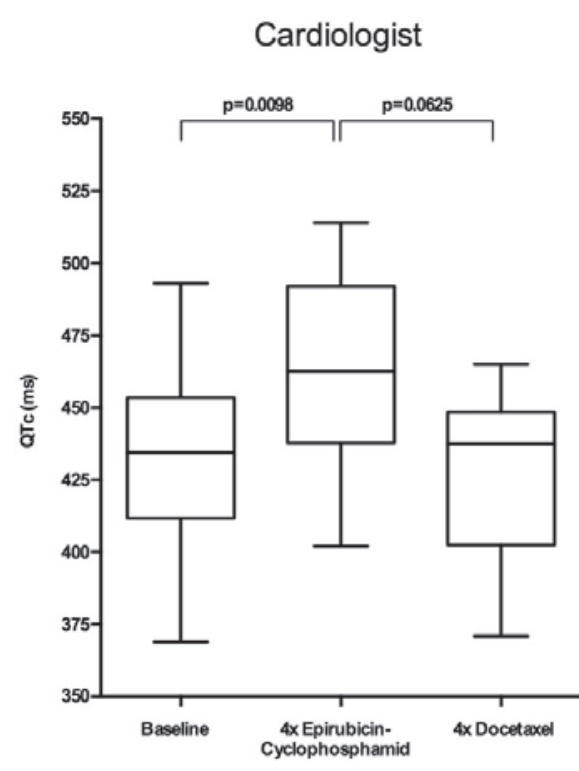

b)

\section{Gynecologist}

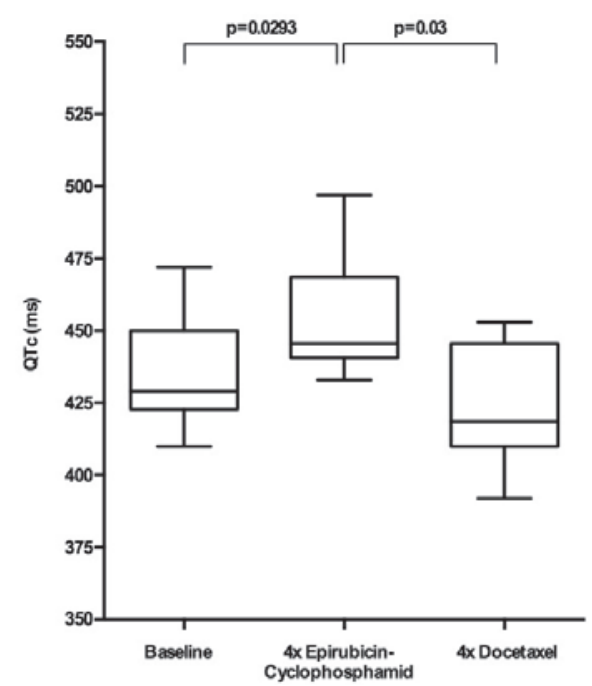

c)

Fig. 1. QTc intervals before and after 4 cycles of chemotherapy with epirubicin and cyclophosphamide (EC) or docetaxel by investigator: a cardiologist, b gynecologist, c computer algorithm.

\section{Statistical Analysis}

The differences in QTc interval were tested with a 2-tailed Wilcoxon signedrank test for paired samples. For multiple comparisons, an ordinary 1-way ANOVA test was applied (GraphPad Prism 6.0, GraphPad Software, Inc., La Jolla, CA, USA). All p values of less than 0.05 were considered significant. For each box plot, the box limits represent the 25th and 75th percentiles and the median, and the whisker ends indicate the 5th and 95th percentiles. Numbers in the text represent the median and minimum-maximum values.

\section{Results}

\section{Patient Demographics}

Between 11/2013 and 07/2014, 10 women with early breast cancer were enrolled in this feasibility study. Descriptive characteristics can be obtained from table 1. All patients had an ECOG performance status of 0 before and after chemotherapy. None of the patients received any medication that can potentially induce QTc interval prolongation, and no further risk factors were found at baseline. No patient had symptoms of cardiac toxicity immediately after treatment. Neither the QTc interval prolongation nor other adverse effects led to discontinuation of chemotherapy.

\section{QTc Interval Prolongation and Interobserver Variability}

To assess the effect of EC-Doc on the QTc interval, records before and after 4 cycles of EC or docetaxel were analyzed. There was a significant QTc interval prolongation after EC treatment independent of the observer (fig. 1). The QTc prolongation (delta QTc) was $28 \mathrm{~ms}$ according to the cardiologist (median 434 (369-493) vs. $462(402-514) \mathrm{ms} ; \mathrm{p}=0.0098), 16 \mathrm{~ms}$ according to the gynecologist (median 429 (410-472) vs. 445 (433-497) ms; p = 0.029), and 24 $\mathrm{ms}$ according to the automated ECG interpretation software (com- puter) (median 414 (384-438) vs. $438(412-464) \mathrm{ms} ; \mathrm{p}=0.0059)$. The QTc interval went back to baseline after 4 cycles of docetaxel (cardiologist: median 437 (371-465) ms, $\mathrm{p}=0.03$; gynecologist: median 418 (392-453) ms, $\mathrm{p}=0.0625$; computer: median 415 (399-440) ms, p = 0.031). When all observer groups were compared by ANOVA test, no significant differences between QTc intervals were detected at baseline $(\mathrm{p}=0.086)$ and after EC $(\mathrm{p}=0.06)$ and docetaxel $(\mathrm{p}=0.8)$, indicating that the interobserver variability is small. Additional ECGs could easily be performed as part of clinical routine without delaying therapy administration.

\section{Biochemical Markers}

For CK-MB and NT-proBNP, only 6 and 8 patients, respectively, were eligible for analysis due to missing blood samples. There was no statistically significant relationship between CK-MB levels and EC or docetaxel administration (median at baseline $(n=6) 15.50(11-79)$ vs. EC $(n=6) 15.00(9-42) \mathrm{U} / \mathrm{l}, \mathrm{p}=0.468$ or docetaxel $(\mathrm{n}=5) 14.00$ (7-23), $\mathrm{p}=0.156)$. Importantly, a statistically significant increase in NT-proBNP was found after EC treatment (median at baseline ( $\mathrm{n}=$ 8) 60 (30-171) ng/l vs. EC $(\mathrm{n}=8) 91$ (40-329) ng/l; $\mathrm{p}=0.0391)$, which declined after docetaxel $(n=5)(38(21-70) n g / l ; p=0.156)$. A clinically relevant elevation above the general cut-off for adults (125 ng/l) was found in 3 patients. 1 patient had elevated NT-proBNP levels of $171 \mathrm{ng} / \mathrm{l}$ at baseline and showed a remarkable increase of up to $329 \mathrm{ng} / \mathrm{l}$ without showing clinical signs of cardiac failure.

\section{Left Heart Systolic and Diastolic Function}

For LVEF analysis, 7 patients could be included. In total, therapy with EC or docetaxel had no significant effect on LVEF levels (median baseline $(\mathrm{n}=7) 70(60-76) \%$ vs. EC $(\mathrm{n}=7) 69(60-76) \%$, $\mathrm{p}=0.671$ or docetaxel $(\mathrm{n}=5) 69(55-69) \%, \mathrm{p}=0.25)$. 


\section{Discussion}

For the treatment of primary breast cancer, an anthracyclinebased regimen is the standard of care $[1,21]$. However, one of the major side effects of the involved drugs is cardiotoxicity. In this study, we found a significant increase in QTc intervals after breast cancer treatment with 4 cycles of EC. In most cases, the increase in QTc interval was within a modest range and did not become clinically relevant. However, a few patients showed QTc intervals of $>470 \mathrm{~ms}$ after treatment, associated with a higher risk of sudden cardiac death $[13,14]$. In comparison to manual analysis, the computer software appears to systematically underestimate the QTc interval. This is in line with previous findings describing a greater variability in manual QT interval measurements compared to automated methods [22]. Nonetheless, the delta QTc time was close to the other observers, indicating a similar trend towards an observerindependent QTc interval prolongation. This is supported by our statistics and a study by Darpo et al. [23] demonstrating that differences between methods disappear when QT changes are analyzed from baseline. Moreover, in another study, the manual interobserver variability of the QTc interval in healthy individuals was found to be close to 0 [24]. Therefore, treating physicians should be able to manually diagnose a possible increase in QTc interval but should be cautious about relying on automated ECG interpretation software.

Therapy with EC-Doc did not induce a significant change in LVEF. Importantly, LVEF measurement shows a low sensitivity in detecting early subclinical signs of cardiotoxicity [25]. This might explain why in our observation period we could not detect any decrease in LVEF despite a significant QTc prolongation. Therefore, echocardiography might be suboptimal for detecting acute cardiac complications.

In the last decade, serum cardiac biomarkers such as troponin, $\mathrm{CK}-\mathrm{MB}$, and natriuretic peptides have been developed to detect cardiotoxicity. In our data set, there was no significant relationship between EC-Doc treatment and CK (data no shown) or CK-MB levels. Interestingly, NT-proBNP levels were significantly increased after EC treatment. This is in line with other studies that showed significant BNP and NT-proBNP elevation after epirubicin-containing chemotherapy $[26,27]$. We observed a decrease in NTproBNP levels after docetaxel administration, indicating that anthracycline-induced cardiac damage can regenerate quickly.

This study is limited by the small number of patients. Moreover, data on docetaxel, serum markers (troponin), and echography (especially parameters of diastolic function) were not available for all patients. In addition, 2 patients received adjuvant treatment with a slightly different dosing schedule, and 4 patients were treated with the anti-HER2/neu antibody trastuzumab. Cardiac toxicity could be more serious when HER2/neu-targeting agents are combined with anthracyclines [28-30]. Our findings, however, are in line with a novel study showing that a combination of docetaxel with trastuzumab has no additional effect on the QT interval [31].

In summary, this data demonstrates that breast cancer treatment with EC causes significant increases in QTc interval and NTproBNP, which are reversible upon docetaxel administration. This indicates that early cardiac dysfunction may already appear during anthracycline-based chemotherapy with a limited long-term effect. In addition, we could show that the interobserver variability in detecting QTc interval prolongation is low, which facilitates the implementation of ECG analysis as part of clinical routine. Further multicenter studies should evaluate the effect on QTc interval prolongation for combination therapies with anthracyclines and novel targeted therapies such as HER2-targeting agents to develop guidelines for the new emerging field of cardio-oncology.

\section{Disclosure Statement}

The authors declare that they have no conflict of interest.

\section{References}

1 Turner N, Biganzoli L, Di Leo A: Continued value of adjuvant anthracyclines as treatment for early breast cancer. Lancet Oncol 2015;16:e362-369.

2 Zagar TM, Cardinale DM, Marks LB: Breast cancer therapy-associated cardiovascular disease. Nat Rev Clin Oncol 2016;13:172-184.

3 Jensen BV, Skovsgaard T, Nielsen SL: Functional monitoring of anthracycline cardiotoxicity: a prospective, blinded, long-term observational study of outcome in 120 patients. Ann Oncol 2002;13:699-709.

4 Altena R, Perik PJ, van Veldhuisen DJ, de Vries EG, Gietema JA: Cardiovascular toxicity caused by cancer treatment: strategies for early detection. Lancet Oncol 2009;10:391-399.

5 Cardinale D, Sandri MT, Martinoni A, Borghini E, Civelli M, Lamantia G, Cinieri S, Martinelli G, Fiorentini C, Cipolla CM: Myocardial injury revealed by plasma troponin I in breast cancer treated with highdose chemotherapy. Ann Oncol 2002;13:710-715.
6 Tian S, Hirshfield KM, Jabbour SK, Toppmeyer D, Haffty BG, Khan AJ, Goyal S: Serum biomarkers for the detection of cardiac toxicity after chemotherapy and radiation therapy in breast cancer patients. Front Oncol 2014;4:277.

7 Selvais PL, Donckier JE, Robert A, Laloux O, van Linden F, Ahn S, Ketelslegers JM, Rousseau MF: Cardiac natriuretic peptides for diagnosis and risk stratification in heart failure: influences of left ventricular dysfunction and coronary artery disease on cardiac hormonal activation. Eur J Clin Invest 1998;28:636-642.

8 Dodos F, Halbsguth T, Erdmann E, Hoppe UC: Usefulness of myocardial performance index and biochemical markers for early detection of anthracycline-induced cardiotoxicity in adults. Clin Res Cardiol 2008;97:318-326.

9 Morris PG, Chen C, Steingart R, Fleisher M, Lin N, Moy B, Come S, Sugarman S, Abbruzzi A, Lehman R, Patil S, Dickler M, McArthur HL, Winer E, Norton L, Hudis CA, Dang CT: Troponin I and C-reactive protein are commonly detected in patients with breast cancer treated with dose-dense chemotherapy incorporating trastuzumab and lapatinib. Clin Cancer Res 2011;17:3490-3499.
10 Allen LaPointe NM, Curtis LH, Chan KA, Kramer JM, Lafata JE, Gurwitz JH, Raebel MA, Platt R: Frequency of high-risk use of QT-prolonging medications. Pharmacoepidemiol Drug Saf 2006;15:361-368

11 Roden DM: Drug-induced prolongation of the QT interval. N Engl J Med 2004;350:1013-1022.

12 Roden DM: Predicting drug-induced QT prolongation and torsades de Pointes. J Physiol 2016;594:2459-2468.

13 Straus SM, Kors JA, De Bruin ML, van der Hooft CS, Hofman A, Heeringa J, Deckers JW, Kingma JH, Sturkenboom MC, Stricker BH, Witteman JC: Prolonged QTc interval and risk of sudden cardiac death in a population of older adults. J Am Coll Cardiol 2006;47:362-367.

14 Johnson JN, Ackerman MJ: QTc: how long is too long? Br J Sports Med 2009;43:657-662.

15 Bagnes C, Panchuk PN, Recondo G: Antineoplastic chemotherapy induced QTc prolongation. Curr Drug Saf 2010;5:93-96.

16 Schwartz CL, Hobbie WL, Truesdell S, Constine LC, Clark EB: Corrected QT interval prolongation in anthracycline-treated survivors of childhood cancer. J Clin Oncol 1993;11:1906-1910.

Interval 
17 Broeyer FJ1, Osanto S, Suzuki J, de Jongh F, van Slooten H, Tanis BC, Bruning T, Bax JJ, Ritsema van Eck HJ, de Kam ML, Cohen AF, Mituzhima Y, Burggraaf J: Evaluation of lecithinized human recombinant super oxide dismutase as cardioprotectant in anthracycline-treated breast cancer patients. Br J Clin Pharmacol 2014;78:950-960.

18 Kitagawa K, Kawada K, Morita S, Inada M, Mitsuma A, Sawaki M, Iino S, Inden Y, Murohara T, Imai T, Ando Y: Prospective evaluation of corrected QT intervals and arrhythmias after exposure to epirubicin, cyclophosphamide, and 5-fluorouracil in women with breast cancer. Ann Oncol 2011;23:743-747.

19 Schijvenaars BJ, van Herpen G, Kors JA: Intraindividual variability in electrocardiograms. J Electrocardiol 2008;41:190-196.

20 Davey P: How to correct the QT interval for the effects of heart rate in clinical studies. J Pharmacol Toxicol Methods 2002;48:3-9.

21 Nitz U, Gluz O, Huober J, Kreipe HH, Kates RE, Hartmann A, Erber R, Scholz M, Lisboa B, Mohrmann S, Möbus V, Augustin D, Hoffmann G, Weiss E, Böhmer S, Kreienberg R, Du Bois A, Sattler D, Thomssen C Kiechle M, Jänicke F, Wallwiener D, Harbeck N, Kuhn W: Final analysis of the prospective WSG-AGO ECDoc versus FEC phase III trial in intermediate-risk (pN1) early breast cancer: efficacy and predictive value of Ki67 expression. Ann Oncol 2014;25:1551-1557.
22 Fosser C, Duczynski G, Agin M, Wicker P, Darpo B: Comparison of manual and automated measurements of the QT interval in healthy volunteers: an analysis of five thorough QT studies. Clin Pharmacol Ther 2009; 86:503-506.

23 Darpo B, Agin M, Kazierad DJ, Layton G, Muirhead G, Gray P, Jorkasky DK: Man versus machine: is there an optimal method for QT measurements in thorough QT studies? J Clin Pharmacol 2006;46:598-612.

24 Dota CD, Edvardsson N, Schützer KM, Olofsson EL, Malm A, Morsing T, Fager G: Inter- and intraday variability in major electrocardiogram intervals and amplitudes in healthy men and women. Pacing Clin Electrophysiol 2003;26:361-366.

25 Swain SM, Whaley FS, Ewer MS: Congestive heart failure in patients treated with doxorubicin: a retrospective analysis of three trials. Cancer 2003;97:2869-2879.

26 Perik PJ, De Vries EG, Boomsma F, van der Graaf WT, Sleijfer DT, van Veldhuisen DJ, Gietema JA: Use of natriuretic peptides for detecting cardiac dysfunction in long-term disease-free breast cancer survivors. Anticancer Res 2005;25:3651-3657.

27 Meinardi MT, van Veldhuisen DJ, Gietema JA, Dolsma WV, Boomsma F, van den Berg MP, Volkers C, Haaksma J, de Vries EG, Sleijfer DT, van der Graaf WT: Prospective evaluation of early cardiac damage induced by epirubicin-containing adjuvant chemotherapy and locoregional radiotherapy in breast cancer patients. J Clin Oncol 2001;19:2746-2753.
28 Fried G, Regev T, Moskovitz M: Trastuzumab-related cardiac events in the treatment of early breast cancer. Breast Cancer Res Treat 2013;142:1-7.

29 Watanabe N, Otsuka S, Sasaki Y, Shimojima R, Wan Y, Uchino K: Cardiac tolerability of concurrent administration of trastuzumab and anthracycline-based regimen as adjuvant chemotherapy for breast cancer. Breast Care (Basel) 2014;9:46-51

30 Du F, Yuan P, Zhu W, Wang J, Ma F, Fan Y, Xu B: Is it safe to give anthracyclines concurrently with trastuzumab in neo-adjuvant or metastatic settings for HER2-positive breast cancer? A meta-analysis of randomized controlled trials. Med Oncol 2014;31:340.

31 Xu N1, Redfern CH, Gordon M, Eppler S, Lum BL Trudeau C: Trastuzumab, in combination with carboplatin and docetaxel, does not prolong the QT interval of patients with HER2-positive metastatic or locally advanced inoperable solid tumors: results from a phase Ib study. Cancer Chemother Pharmacol 2014;74:12511260 . 